\title{
Profiling Sea Ice with a Multiple Altimeter Beam Experimental Lidar (MABEL)
}

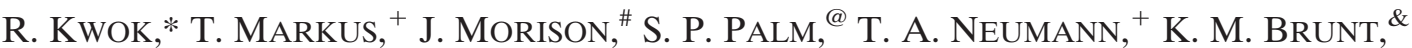 \\ W. B. COOK, ${ }^{* *}$ D. W. HANCOCK, ${ }^{++}$AND G. F. CunNINGHAM ${ }^{*}$ \\ * Jet Propulsion Laboratory, California Institute of Technology, Pasadena, California \\ ${ }^{+}$Cryospheric Sciences Laboratory, NASA Goddard Space Flight Center, Greenbelt, Maryland \\ \# Polar Science Center, University of Washington, Seattle, Washington \\ ${ }^{@}$ Science Systems and Applications, Inc., Lanham, Maryland \\ ${ }^{\&}$ Cryospheric Sciences Laboratory, and GESTAR, NASA Goddard Space Flight Center, Greenbelt, Maryland \\ ** Mesoscale Atmospheric Processes Laboratory, NASA Goddard Space Flight Center, Greenbelt, Maryland \\ ${ }^{++}$NASA Wallops Flight Facility, Wallops Island, Virginia
}

(Manuscript received 20 May 2013, in final form 20 December 2013)

\begin{abstract}
The sole instrument on the upcoming Ice, Cloud, and Land Elevation Satellite (ICESat-2) altimetry mission is a micropulse lidar that measures the time of flight of individual photons from laser pulses transmitted at $532 \mathrm{~nm}$. Prior to launch, the Multiple Altimeter Beam Experimental Lidar (MABEL) serves as an airborne implementation for testing and development. This paper provides a first examination of MABEL data acquired on two flights over sea ice in April 2012: one north of the Arctic coast of Greenland and the other in the east Greenland Sea. The phenomenology of photon distributions in the sea ice returns is investigated. An approach to locate the surface and estimate its elevation in the distributions is described, and its achievable precision is assessed. Retrieved surface elevations over relatively flat leads in the ice cover suggest that precisions of several centimeters are attainable. Restricting the width of the elevation window used in the surface analysis can mitigate potential biases in the elevation estimates due to subsurface returns at $532 \mathrm{~nm}$. Comparisons of nearly coincident elevation profiles from MABEL with those acquired by an analog lidar show good agreement. Discrimination of ice and open water, a crucial step in the determination of sea ice freeboard and the estimation of ice thickness, is facilitated by contrasts in the observed signal-background photon statistics. Future flight paths will sample a broader range of seasonal ice conditions for further evaluation of the year-round profiling capabilities and limitations of the MABEL instrument.
\end{abstract}

\section{Introduction}

NASA's second-generation Ice, Cloud, and Land Elevation Satellite (ICESat-2), currently planned for launch in late 2016, will provide observations to quantify the changes in ice sheets and sea ice, and key insights into their behavior (Abdalati et al. 2010). To achieve this, precise laser measurements of surface elevation, building on the capabilities of its predecessor (ICESat-1), will be acquired to assess ice sheet mass balance and processes, as well as the time-varying thickness and volume of sea ice in the Arctic and Southern Oceans. ICESat-2 will also measure sea surface height in the icefree sub-Arctic seas and provide large-scale vegetation

Corresponding author address: Ron Kwok, Jet Propulsion Laboratory, California Institute of Technology, 4800 Oak Grove Dr., Pasadena, CA 91109.

E-mail: ron.kwok@jpl.nasa.gov biomass estimates through the measurement of vegetation canopy height. Combining data from the ICESat-2 mission with existing and forthcoming altimetry datasets will yield a $15+$ year record of elevation change.

Instead of the analog lidar used for ICESat-1 (Abshire et al. 2005; Schutz et al. 2005), ICESat-2 will employ a photon-counting approach to obtain better measurement sensitivity with lower resource (power) demands on the satellite platform. A high repetition rate $(10 \mathrm{kHz})$, low pulse energy laser at $532 \mathrm{~nm}$ in conjunction with sensitive detectors will measure the range of individual photons scattered from the surface. Six across-track beams will profile the ice surface, and for ice sheets the multiple beams address the need for unambiguous separation of ice sheet slope from elevation changes (Zwally et al. 2011).

Photon-counting (PC), or micropulse, lidars have been employed for many years in atmospheric (e.g., cloud and aerosol) sounding applications (Spinhirne

DOI: 10.1175/JTECH-D-13-00120.1 
1993; McGill et al. 2002), but the use of this type of lidar for high-precision surface altimetry is relatively new (e.g., Harding et al. 2011). Whereas traditional altimeters have used analog detection with fast digitization of the return waveform, PC lidars provide the distance traveled by individual photons. Surface elevations are estimated from the observed photon distributions, sometimes referred to as "photon clouds." PC implementations reduce the thermal and power demands for a satellite-based instrument, but a small field of view is required to limit the confounding effects of solar background in the return data. For ICESat-2, the diameter of each laser footprint on the surface is $\sim 10 \mathrm{~m}$ (compared to the ICESat footprint of $\sim 70 \mathrm{~m}$ ) with a receiver field of view of $\sim 40-\mathrm{m}$ diameter. The design uses six transmit beams in three pairs in a rectangular configuration. A yaw angle from the satellite track then sets the specific spacing of the footprints (http://icesat.gsfc.nasa.gov/ icesat2).

The Multiple Altimeter Beam Experimental lidar (MABEL) is an airborne PC lidar used as a technology demonstrator for the photon-counting instrument [Advanced Topographic Laser Altimeter System (ATLAS)] on the ICESat-2 mission (McGill et al. 2013). Data from this instrument will be used to validate instrument models and to assess performance of ATLAS that will be launched on ICESat-2. Table 1 compares the performance of ICESat-2 and MABEL. The first Arctic deployment of MABEL in April 2012 acquired sea ice data with two dedicated flight paths, one covering $>3000 \mathrm{~km}$ in length (8 April) and the other $>1000 \mathrm{~km}$ (10 April) (Fig. 1). These survey paths were selected to provide a broad sampling of ice conditions prior to the expected onset of melt over the Arctic sea ice cover. The first ER-2 flight on 8 April (operations flown out of Keflavik, Iceland) acquired data over the Lincoln Sea-the compact, deformed ice north of the coast of Greenland-and the sea ice in the Fram Strait and east Greenland Sea between Greenland and Iceland. The second flight resurveyed the track just east of the Greenland coast (see Fig. 1). On 10 April, the ER-2 was joined by the P-3 from Operation IceBridge (Koenig et al. 2010). The P-3 carried the Airborne Topographic Mapper (ATM) instrument (Krabill et al. 2002), an analog lidar, to provide near-coincident coverage for assessment of MABEL's performance.

Since this is the first acquisition of this type of $\mathrm{PC}$ data over Arctic sea ice, the objective of this paper is to investigate the phenomenology and to examine the utility and achievable precision of the MABEL instrument for retrieval of ice and open water elevations for freeboard calculations (e.g., in Kwok et al. 2009). The intent is to
TABLE 1. Comparison of MABEL and ICESat-2 instrument parameters and expected-observed performance.

\begin{tabular}{|c|c|c|c|}
\hline Parameter & ICESat-2 & \multicolumn{2}{|c|}{$\begin{array}{c}\text { MABEL-Channel } 6 \\
\text { April } 2012\end{array}$} \\
\hline Operational altitude, $R(\mathrm{~km})$ & 490 & \multicolumn{2}{|c|}{20} \\
\hline Wavelength, $\lambda(\mathrm{nm})$ & 532 & \multicolumn{2}{|c|}{532} \\
\hline Telescope diameter, $A_{T}$ & $0.8 \mathrm{~m}$ & \multicolumn{2}{|c|}{$12.7 \mathrm{~cm}$} \\
\hline PRF & $10 \mathrm{kHz}$ & \multicolumn{2}{|c|}{$5 \mathrm{kHz}$} \\
\hline Pulse energy, $E_{t}(\mu \mathrm{J})$ & $41 / 160^{\mathrm{a}}$ & \multicolumn{2}{|c|}{$1.4 / 0.2^{\mathrm{b}}$} \\
\hline Footprint $\left(1 / e^{2}\right)(\mathrm{mrad})(\mathrm{m})$ & $31(15)$ & \multicolumn{2}{|c|}{$100(2)$} \\
\hline FOV $(\mathrm{mrad})(\mathrm{m})$ & $83(41)$ & \multicolumn{2}{|c|}{$210(4.2)$} \\
\hline Filter width (p.m.) & 30 & \multicolumn{2}{|c|}{$\sim 150$} \\
\hline $\begin{array}{l}\text { System-detector efficiency, } \\
\qquad T_{o} Q_{E}(\%)\end{array}$ & 3 & \multicolumn{2}{|c|}{5} \\
\hline Swath width $(\mathrm{km})$ & \pm 3.00 & \multicolumn{2}{|c|}{ \pm 1.05} \\
\hline $\begin{array}{l}\text { Signal levels }{ }^{\mathrm{c}} \text { over winter sea } \\
\text { ice (photons per shot) }\end{array}$ & Expected & Expected $^{\mathrm{b}}$ & Observed $^{\mathrm{c}}$ \\
\hline $\begin{array}{l}\text { Snow-covered ice } \\
\quad(\text { albedo }=0.9)\end{array}$ & $1.6 / 6.2^{\mathrm{a}}$ & $1.4 / 0.19^{b}$ & $0.2^{\mathrm{d}}$ \\
\hline Open lead (albedo $=0.15)$ & $0.26 / 1.0^{\mathrm{a}}$ & $0.2 / 0.03^{\mathrm{b}}$ & $0.04^{\mathrm{d}}$ \\
\hline
\end{tabular}

${ }^{\mathrm{a}}$ Weak/strong beam.

${ }^{\mathrm{b}}$ Before/after fiber damage.

${ }^{\mathrm{c}}$ Lidar equation used to compute expected signal levels: $N_{r}=\alpha_{\lambda}\left(E_{t} \lambda / \hbar c\right)\left(T_{A} A_{T} T_{o} Q_{E} / \pi R^{2}\right)$, where $\alpha_{\lambda}=$ surface albedo, $T_{A}=$ molecular atmospheric transmission ( 0.81 is used in calculations), $Q_{E}=$ detector quantum efficiency, $T_{o}=$ system optical transmission, $\mathrm{c}=$ speed of light, and $\hbar=$ Planck's constant.

${ }^{\mathrm{d}}$ Observed signal levels after fiber damage (see sections 3 and 4).

provide an examination of MABEL acquisitions over sea ice but not a comprehensive analysis of the PC instrument for surface profiling. The paper is organized as follows. Section 2 describes briefly the MABEL instrument, its data products, and the ATM acquisitions used in our analysis. In section 3, we examine the phenomenology of photon returns from the sea ice cover with segments of MABEL data acquired during this Arctic deployment. An approach to locate the surface and estimate its elevation in the photon clouds is described in section 4 . The achievable precision and quality are assessed over several large leads. In section 5, we compare the retrieved elevations from MABEL with two near-coincident profiles from the ATM lidar. Summary remarks and conclusions are provided in the last section.

\section{Data description}

In this section, we provide brief descriptions of the two lidar systems (MABEL and ATM) and their elevation data products used in this paper. MABEL datasets and documentation are available online (http:// icesat.gsfc.nasa.gov/icesat $2 / \mathrm{data} / \mathrm{mabel} / \mathrm{mabel}$ docs. php). Datasets from Operation IceBridge (OIB) are 

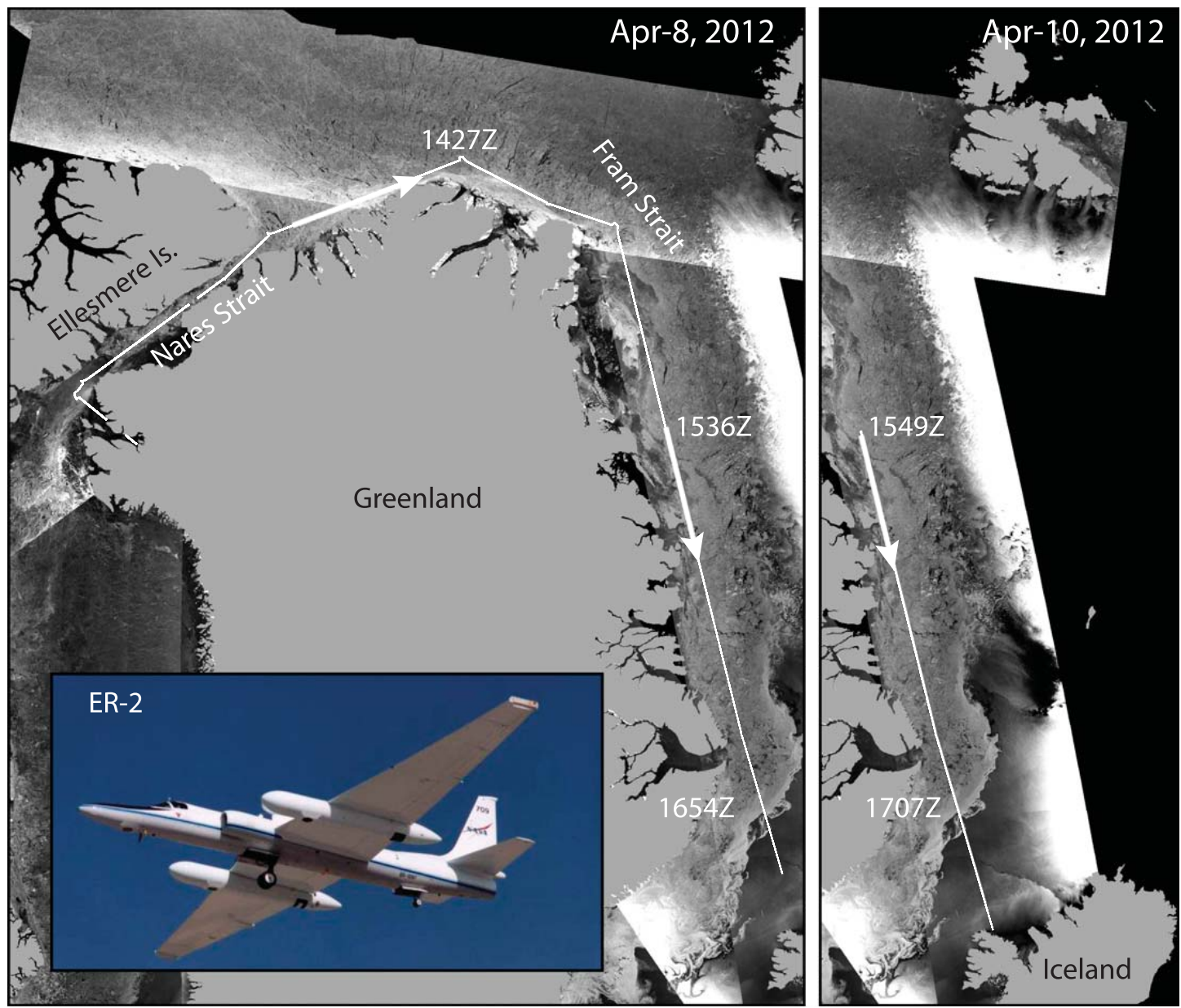

FIG. 1. MABEL flight paths (on 8 and $10 \mathrm{Apr}$ ) plotted on an Environmental Satellite (Envisat) synthetic aperture radar (SAR) mosaic of the region acquired on 8 Apr. Near-coincident ATM data from OIB were acquired on 10 Apr. Inset shows the ER-2 aircraft. MABEL is located in the nose of the platform. The segments discussed in the paper are from flight paths south of the Fram Strait.

archived at the National Snow and Ice Data Center (NSIDC). In particular, the dataset of interest in this paper is the surface elevation data acquired by the Airborne Topographic Mapper (ATM), an analog lidar used to provide high-precision lidar mapping of surface elevation.

\section{a. MABEL-Photon-counting lidar}

For this first deployment to the Arctic, the MABEL instrument was mounted in the nose of the ER-2 aircraft. The relevant instrument parameters are shown in Table 1. The MABEL laser has a pulse width of $\sim 2 \mathrm{ns,}$ and the pulse repetition frequency (PRF) can be varied between 5 and $25 \mathrm{kHz}$. With a PRF of $5 \mathrm{kHz}$ (used during these flights) and a nominal ER-2 ground speed of $\sim 200 \mathrm{~m} \mathrm{~s}^{-1}$, a pulse is transmitted approximately every $4 \mathrm{~cm}$ along track. From an operating altitude of $20 \mathrm{~km}$, the telescope field of view (FOV: $\sim 210 \mu \mathrm{rad}$ ) covers a $\sim 4$-m-diameter footprint on the ground and the laser illuminates a smaller spot of $\sim 2 \mathrm{~m}\left(1 / e^{2}\right)$ in diameter; this ensures that the illuminated spot is within the FOV of the telescope. An inertial measurement unit (IMU) is mounted directly on the telescope assembly to permit accurate determination of instrument pointing. A digital camera system (DCS) with a resolution of $\sim 1 \mathrm{~m}$ captures aerial images of the surface along the MABEL tracks. These images are invaluable for identifying the surface types (open water, snow-covered ice, thin ice, etc.) sampled by the lidar.

The output of the MABEL laser at 1064 and $532 \mathrm{~nm}$ is split into eight $1064-\mathrm{nm}$ and sixteen 532-nm beams. Returns from the two wavelengths facilitate the examination of elevation biases due to subsurface scattering from water, and ice and snow volumes. In MABEL, each beam path can accommodate a small neutral density filter for varying the energy levels at each footprint. Coupling a given beam to one of 107 fibers (at each wavelength), which are pointed at a fixed set of look 
angles, allows selectable off-nadir sampling to maximum view angles of $\pm 3^{\circ}( \pm 52 \mathrm{mrad})$. From a flight altitude of $20 \mathrm{~km}$, the beams are capable of acquiring data to $\pm 1.05 \mathrm{~km}$ on each side of the track (see Fig. 2 in McGill et al. 2013). With a separate set of transmitter fibers for each wavelength, the same surface area can be illuminated with both wavelengths (i.e., the footprints for each wavelength can overlap). For the two flights over sea ice of interest here, MABEL was configured with a narrow measurement swath. The 532-nm channels were set to illuminate the following look angles (relative to nadir) at approximately $0, \pm 0.1, \pm 0.2, \pm 1.0, \pm 1.9, \pm 2.0, \pm 3.0$, \pm 4.0 , and $\pm 5.0 \mathrm{mrad}$. The $1064-\mathrm{nm}$ channels were set at $\pm 0.1, \pm 0.2, \pm 1.9$, and $\pm 2.0 \mathrm{mrad}$. At the $20 \mathrm{-km}$ measurement altitude, this provided a cross-track width of $\pm 100 \mathrm{~m}$.

The sixteen 532-nm receiver fibers are routed to a 16-channel Hamamatsu model H7260 photomultiplier tube (PMT) detector and the eight 1064-nm receiver fibers are routed to eight individual Excelitas single-photoncounting modules (SPCMs). For these detectors, there is a dead time $\left(t_{d}\right)$ of $\sim 2.5 \mathrm{~ns}$. Detector dead time is time required for the detector to recover from a photon event (the triggering of the detector) before the next photon can be detected. For MABEL, the surface return and background rates are much lower than $400 \mathrm{MHz}\left(1 / t_{d}\right)$; thus, an insignificant fraction of photons events occur during the dead time, so the sampled rate is linear with the input rate. Detected photons are time tagged for range determination and have a measured resolution of 83 ps (about $12 \mathrm{~mm}$ ).

Analyzed MABEL products (release 7) contain geolocated elevations of individual photons between -1 and $5 \mathrm{~km}$ relative to the World Geodetic System 84 (WGS84) ellipsoid, a commonly used datum for measurement of elevations. Products are distributed as data files, each containing $60 \mathrm{~s}$ of surface returns from 300000 pulses, covering $\sim 13 \mathrm{~km}$ along track. Henceforth, we refer to these as MABEL file segments.

\section{b. MABEL instrument performance}

An unexpected physical deterioration of optical fibers in the transmit path over the course of this deployment affected each beam differently. The deterioration was due to damage sustained during the alignment of the laser and beam splitter prior to the beginning of the flights. This resulted in an overall degradation in instrument performance and data quality because of reductions in transmitted energy and received signal strength (i.e., reduced photon count from the surface). Prior to fiber damage, the MABEL signal levels were expected to be 1.4 and 0.2 photons per shot (see Table 1 ) over a highreflectance snow-covered sea ice surface (albedo: $\sim 0.9$ ) and water in open leads (albedo: $\sim 0.15$ ), respectively; the goal was to match the per-shot signal performance of the ICESat-2 instrument.

To assess the impact of the fiber damage on signal levels, we selected one beam (beam 6) at the center of the array with the best signal strength. Based on our estimates, the fiber damage caused at least a 7-times reduction in pulse energy (from 1.4 to $0.2 \mu \mathrm{J}$ ) with a similar reduction in the received signal level. Expected per-shot photon count decreased from 1.4 to 0.19 for snow-covered sea ice and from 0.2 to 0.03 for open water leads (see Table 1). These estimates can be compared with the observed returns of 0.2 and 0.04 photon per shot from these two surface types (see Table 1; also discussed in sections 3 and 4). The near correspondence between the expected and observed signal levels are probably fortuitous because there are uncertainties in the albedo of the surfaces that we selected for comparisons.

Even though the signal levels are reduced, signal photons from consecutive MABEL shots can be combined to simulate the signal levels of ICESat-2. However, there are two considerations if that approach were used: first photon bias and background counts. Since the average surface signal per shot from high reflectance surfaces in the current MABEL dataset is less than one, the first photon bias in surface elevation estimation (described in Yang et al. 2011) is zero. This is good in practice, but this does not allow us to examine the impact of first photon bias in sea ice altimetry. Second, when multiple MABEL shots are combined to simulate the signal from a single ICESat-2 shot, the effective background rate (per shot) increases linearly. There are $\sim 17$ MABEL shots (pulse interval: $\sim 4 \mathrm{~cm}$ ) in an ICESat-2 pulse interval $(70 \mathrm{~cm})$. If 17 shots were combined, a MABEL background rate of $0.1 \mathrm{MHz}$ (estimated in section 3) would give an effective rate of $1.7 \mathrm{MHz}$. This is, in fact, close to what one expects to see in ICESat-2 data from the polar regions. Also of note is that the degraded MABEL signal density (i.e., counts per meter) of $1.0-5.0$ photons $\mathrm{m}^{-1}$ over leads/snow-covered ice is roughly equivalent to the expected ICESat-2 signal density of $1.4 / 8.6$ photons $\mathrm{m}^{-1}$ over these same surfaces. In any case, these are important factors to consider when examining this particular dataset.

\section{c. ATM lidar}

For a more detail description of the ATM instrument, the reader is referred to Krabill et al. (2002). Briefly, the ATM is a conical-scanning laser ranging system operated at a wavelength of $532 \mathrm{~nm}$ with a pulse repetition frequency of $5 \mathrm{kHz}$ and a scan rate of $20 \mathrm{~Hz}$; the off-nadir scan angle is $15^{\circ}$. To provide accurate elevation estimates, 
the instrument includes a differential GPS system for accurate positioning of the aircraft, and inertial sensors (accelerometers and gyroscopes) for precise attitude determination. With nominal OIB flight parameters (i.e., operating altitude and ground speed: $500 \mathrm{~m}$ and $250 \mathrm{kt} ; 1 \mathrm{kt}=0.51 \mathrm{~m} \mathrm{~s}^{-1}$ ), the ATM scanning geometry provides an across-track scan swath of $\sim 250 \mathrm{~m}$. Near the center of the swath, the spacing between neighboring laser footprints, of $\sim 1 \mathrm{~m}$ in diameter, is approximately $3-4 \mathrm{~m}$ in the along- and across-track directions. The sample density is higher (submeter) near the edges of the swath due to the conical scanning geometry of the system. We note here that this lidar does not provide contiguous spots on the ground.

Generally, the backscatter from a snow or ice surface within the beam of the ATM is sufficient for measurement of the time delay of a return signal and for determination of the total propagation distance. However, the presence of extremely smooth surfaces along the flight path results in some measurement dropouts, due to weak surface returns at off-nadir angles. In the processing of the data, the travel time data are combined with GPS navigation measurements and aircraft orientation parameters to derive surface elevation measurements relative to the WGS84 reference ellipsoid. Typical elevation accuracy is better than $10 \mathrm{~cm}$ (Krabill et al. 2002). Retrieved elevations are provided in data files that cover tracks of $\sim 35 \mathrm{~km}$ in length, each containing over a million elevation estimates.

\section{Phenomenology}

To carry out high-precision surface profiling of the sea ice cover with the PC approach, a first step is to understand the expected returns from various surface types and contributing noise sources (system and geophysical). Even though the physical basis of the lidar returns from the surface are not different from that of an analog lidar, there are important distinctions and considerations in the use of these data. With several examples in this section, we show the signatures of sea ice returns within an elevation window close to the surface. As mentioned above, all the MABEL data used here are from channel $6(532 \mathrm{~nm})$. Since the dataset was acquired in early April, the sea ice conditions can be considered to be that of early spring; that is, there is very little surface melt except perhaps near the ice edge. Prior to melt, a significant fraction of Arctic sea ice is snow covered (of high albedo), while the remaining areas are covered by newly formed thinner ice (of lower albedo) that have yet to acquire a snow cover and by open water. Significant contrasts between these surfaces are expected.

\section{a. Signal versus noise photons}

One distinction between PC and analog lidars is the discrete nature of the noise. The total number of photons $\left(\mathrm{NP}_{\text {tot }}\right)$ within a range window includes those backscattered from the surface $\left(\mathrm{NP}_{\text {surf }}\right)$ and atmosphere $\left(\mathrm{NP}_{\mathrm{atm}}\right)$, those from solar background $\left(\mathrm{NP}_{\mathrm{Sbkg}}\right)$, and those introduced by noise in the detector $\left(\mathrm{NP}_{\mathrm{det}}\right.$ : dark counts), namely,

$$
\mathrm{NP}_{\text {tot }}=\mathrm{NP}_{\text {surf }}+\left(\mathrm{NP}_{\mathrm{atm}}+\mathrm{NP}_{\text {Sbkg }}+\mathrm{NP}_{\text {det }}\right) \text {. }
$$

Clearly, the larger the fraction of surface (or signal) photons compared to those photons (background) from sources within the parentheses (i.e., higher signal-tonoise ratio), the better for surface retrieval. Background or noise photons add to uncertainties in the surface profiling and retrieval processes. On a cloud-free day, $\mathrm{NP}_{\mathrm{atm}}$ can be considered to be small compared to the other terms. For MABEL, the detector noise rate (at $0.02 \mathrm{kHz}$ ) is much smaller than the signal rates and solar background discussed below. Henceforth, $\mathrm{NP}_{\mathrm{atm}}$ and $\mathrm{NP}_{\text {det }}$ will be assumed to be negligible and not discussed.

The solar background rate ( $B_{s}$, usually measured in photons per second or $\mathrm{MHz}$ ) is the solar zenith radiance due to surface and atmospheric scattering of solar energy at the laser wavelength $(\lambda)$ that is detected by the instrument. The solar zenith angle varies latitudinally, seasonally, and with time of day. The quantity $B_{S}$ is negligible at night but significant when the sun is high in the sky. Neglecting atmospheric effects, this quantity can be calculated as

$$
B_{s}=S_{\lambda} \alpha_{\lambda} \cos \left(\theta_{z}\right)(\mathcal{S})(\mathrm{MHz}) .
$$

In the equation, $B_{s}$ is dependent on the bidirectional reflectance of the surface $\left(\alpha_{\lambda}\right)$, the solar zenith angle $\left(\theta_{z}\right)$, and the solar flux at the top of the atmosphere $\left(S_{\lambda}\right)$. The quantity $\mathcal{S}$ includes constants and system parameters that are not germane to the current discussion. This calculated background rate (in $\mathrm{MHz}$ ) when divided by $0.5 \times$ (speed of light) gives the expected number of background photons found within a 1-m elevation window.

The observed and expected [using $\alpha_{\lambda}=0.8$ in Eq. (1)] solar background rates along the two flight paths are shown in Fig. 2. The observed background rate is calculated using the total number of photons between an elevation of 4 and $5000 \mathrm{~m}$ above the surface in each MABEL file segment (or 300000 shots). The observed background rates are less than expected, but for long stretches of the $3000-\mathrm{km}$ flight line on 8 Apr north of the 
a) Apr 8, 2012

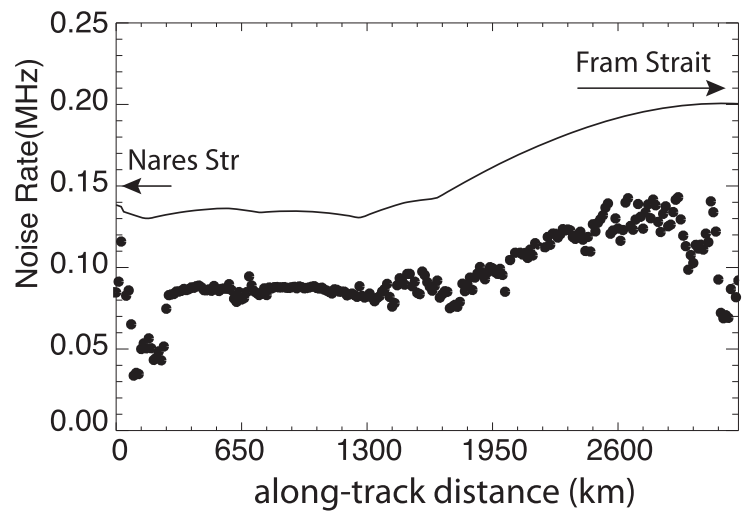

b) Apr 10, 2012

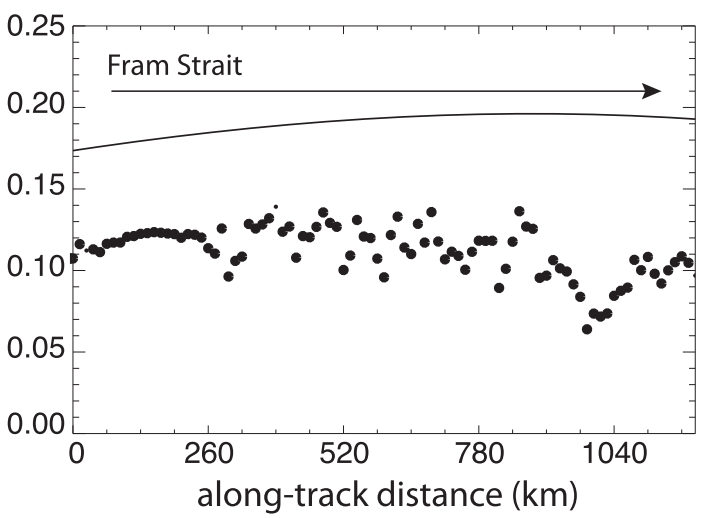

FIG. 2. Calculated (black line) and observed (solid circles) solar background noise rates over flight paths on (left) 8 and (right) 10 Apr. See Fig. 1 for location of these flight paths.

Greenland coast, the calculated and observed background noise rates are highly correlated. There is little variability in albedo in this region of relatively compact snow-covered sea ice, and the changes in the solar zenith angle explain a large fraction of the variability in the solar background rates. This can be compared to the more variable rates over the mixed ice cover in Nares Strait and those just south of Fram Strait. Here, the mix of ice types from old ice to that of recently opened leads, with large contrasts in albedos, contributes to the observed variability. Similar variability can be found in the shorter flight line on 10 April (a repeat survey of the track flown on 8 April) of the mixed ice cover (deformed with relatively wide leads) just south of Fram Strait. The discrepancy between the calculated and observed solar background is due to unmodeled changes in albedo and to residual biases in system parameters that are currently being addressed by the MABEL instrument team.

For the two flight paths, the magnitude of $B_{s}(\sim 0.1 \mathrm{MHz})$ translates into an approximate mean noise density of $\sim 3.3 \times 10^{-4}$ photons $^{-1}$. This is equivalent to $1.5 \times$ $10^{-3}$ photons over a limited elevation window containing the surface (say, $5 \mathrm{~m}$ ). The likelihood of finding a noise photon is relatively small compared to the number of expected surface (or signal) photons of between 0.1 and 1.0 photon per shot from a dark sea ice lead or a snow-covered ice surface (see discussion below). It should also be noted that the detector dead time is much smaller (by three orders of magnitude) than the expected interval between background photons $\left(1 / B_{s}\right)$; thus, the impact of the background photons on the surface elevation retrieval is small.

More importantly for sea ice, since $B_{s}$ provides a measure of surface albedo, it is a useful parameter for supporting the classification of surface types in the photon data (see discussion in next section).

\section{b. Sea ice in two MABEL file segments}

We use two examples (see Figs. 3 and 4) to show the signatures of different surface types in the geolocated photon clouds from MABEL. Of particular geophysical interest are the contrasts in the signal and background rates for discrimination of ice and open water in the retrieval of sea ice freeboard. Each example shows a $5.5-\mathrm{km}$ subsegment extracted from a $13-\mathrm{km}$ file segment. A large open water lead is centered in the first example (Fig. 3) while more along-track spatial structure in the ice cover is seen in the second example (Fig. 4).

The DCS imagery (in Figs. 3a and 4a) shows the sea ice cover profiled by MABEL. Both examples contain at least four sea ice types as suggested by their image intensities and elevations (relative to the dark lead). Visually, the brightest samples seem to belong to that of snow-covered sea ice; the next brightest is thin ice with a thin layer of snow; then thin ice that is bare of snow; and last, of open water in leads. We recognize the thin ice categories as thin ice because their elevations are closest to that of open water (see Figs. 3c-e and Figs. 4c-e). The derivation of surface elevation estimates shown in Figs. $3 \mathrm{e}$ and $4 \mathrm{e}$ are discussed in the next section.

The red and black dots (in Figs. $3 \mathrm{~b}$ and $4 \mathrm{~b}$ ) show the along-track surface ( -3 to $4 \mathrm{~m}$ ) and background noise (4 to $5000 \mathrm{~m}$ ) counts, respectively-henceforth, the following notation is used to describe elevation intervals: $[-3,4] \mathrm{m}$ and $[4,5000] \mathrm{m}$. In both examples, the profile of the background counts is positively correlated to the intensities of the DCS samples $(\rho \sim 0.98)$. This is expected as the PC system is similar to a camera system that records scattering of incident solar flux at $532 \mathrm{~nm}$. The ratio of background $(B)$ photon counts $\mathrm{NP}_{\text {snow }}^{B} / \mathrm{NP}_{\text {lead }}^{B}$ from snow-covered ice surfaces and open water leads are of $\sim 5$-approximates what one expects from the albedo 


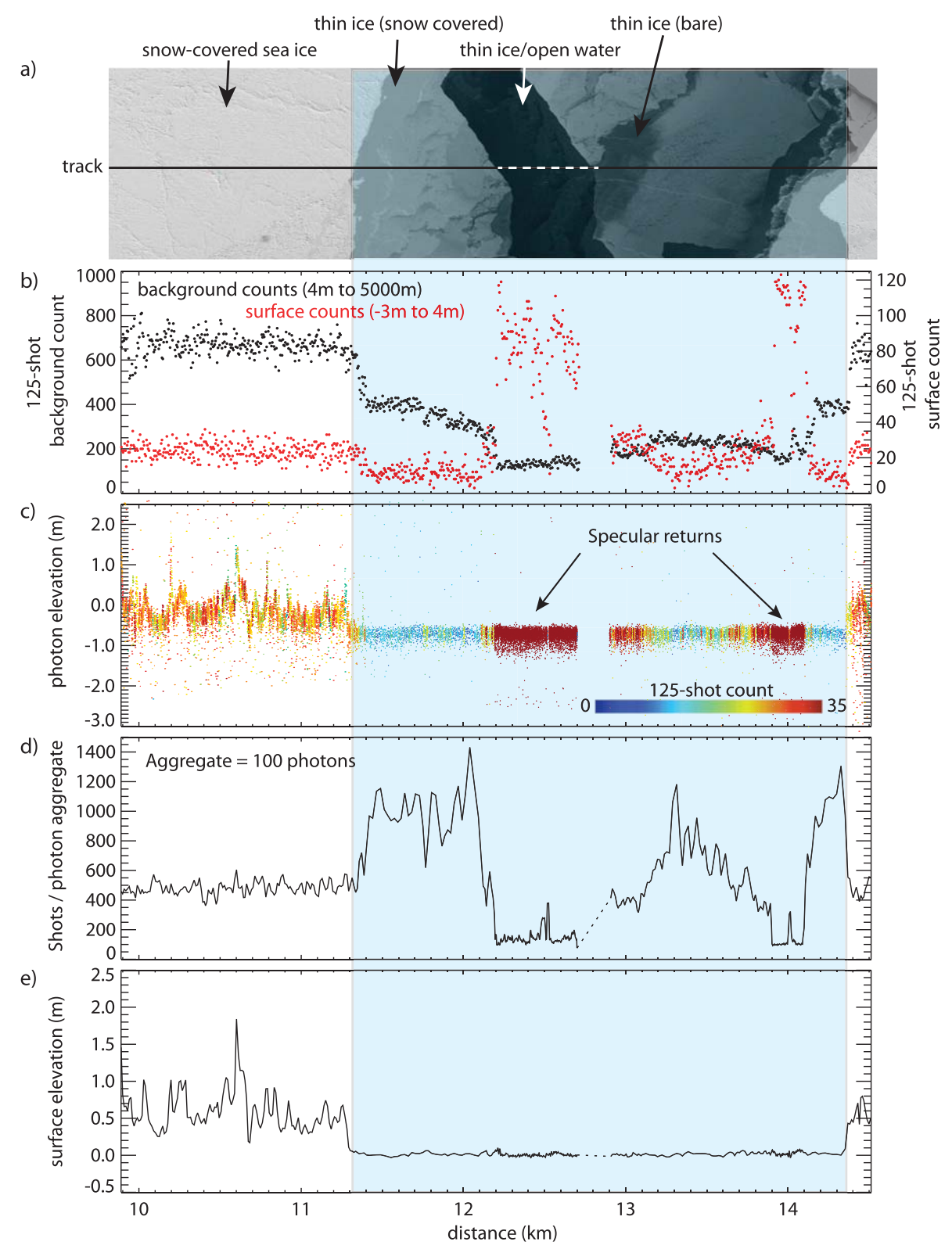

FIG. 3. Returns from the sea ice cover (channel 6) along a cloud-free $~ 5.5$-km MABEL track. (a) DCS image containing the MABEL track. (b) Total photon counts from two elevation intervals: surface ( -3 to $4 \mathrm{~m}$ ) and background ( $4-5000 \mathrm{~m}$ ); photon counts are sums of returns from 125 shots ( $\sim 5 \mathrm{~m}$ along track); note the different scales for the (left) background and (right) surface (right) counts. (c) Photon elevations; colors represents total counts for 125 shots. (d) Number of shots needed to aggregate at least 100 signal photons. (e) Retrieved elevation. Location of this MABEL segment is shown in Fig. 1. Transparent light blue band shows characteristics of photons originating near the sea surface.

differences [of $\sim 0.8$ for ice and $\sim(0.1-0.2)$ for leads] between the two surface types (Grenfell and Perovich 1984; Perovich et al. 2002).

In these examples, the surface or signal counts and the DCS image intensities are only correlated over the brighter surface categories of snow-covered sea ice, snowcovered thin ice, and thin ice. Over the dark sea ice leads with low background counts, it is striking that the signal or surface photon counts are nearly 5-6 times the surface counts from a snow-covered ice surface (see Figs. $3 b$ and $4 \mathrm{~b}$ ). This is contrary to what one expects from a Lambertian surface, and it suggests that these high signal counts are associated with near-nadir specular returns from very smooth surfaces in the open lead. This contrast in surface photon density can be seen in the color-coded plot of photon elevations in Figs. $3 c$ and 4c, 

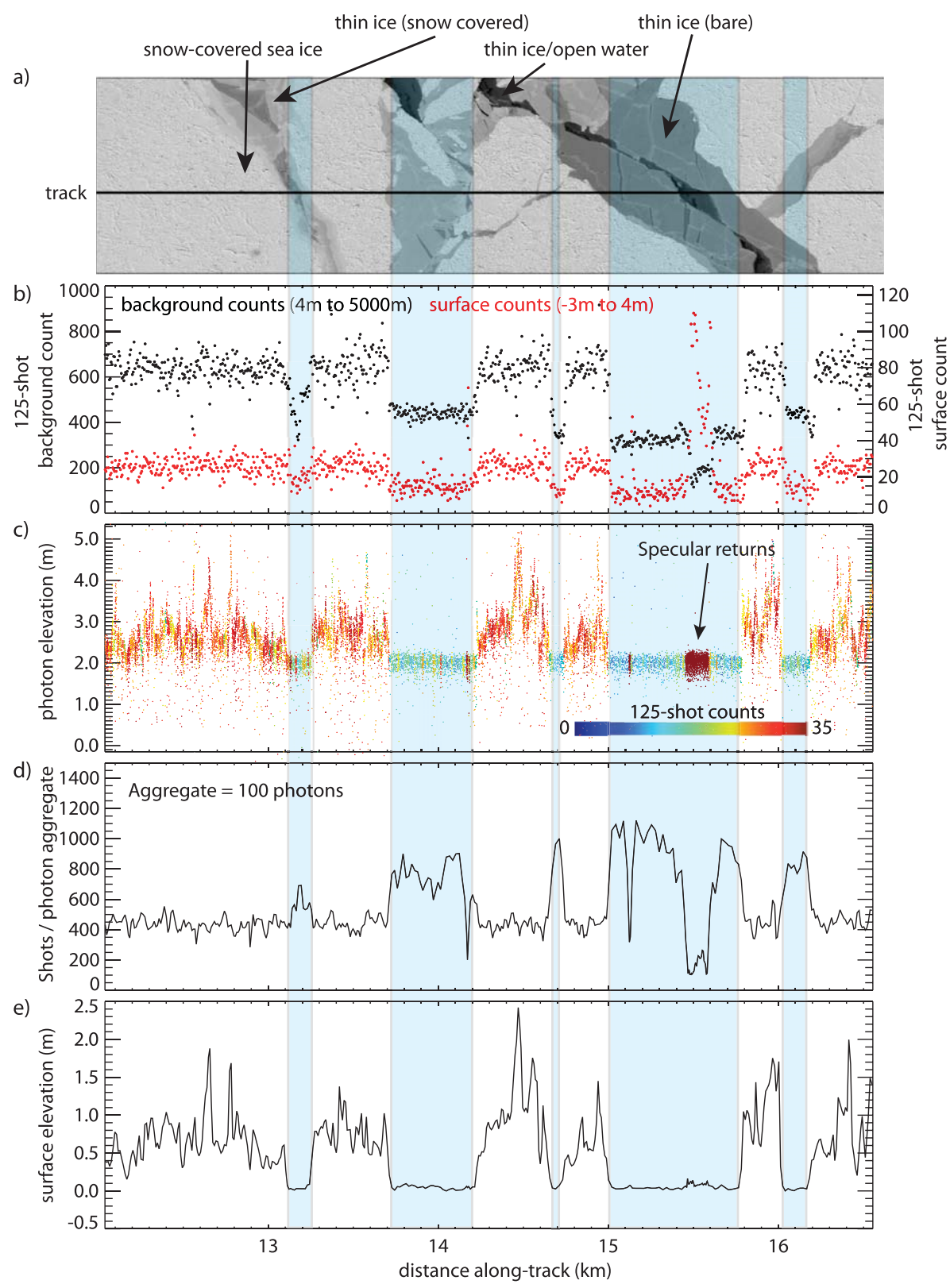

FIG. 4. As in Fig. 3, but for a different segment.

where the colors represent 125 -shot photon counts in the neighborhood (centered) of each detected photon. How close to nadir do these returns originate? From consideration of the attitude and relative alignment of the IMU and the lidar, the returns seen in this and examples that follow are within $1^{\circ}-2^{\circ}$ of nadir.

Away from the specular returns, the ratios of surface $(S)$ photon counts $\mathrm{NP}_{\text {snow }}^{B} / \mathrm{NP}_{\text {thinice }}^{B}$ between the snowcovered ice surface and thin ice in both the solar background $(B)$ are $\sim(3-4)$. As expected the contrast is somewhat lower than the albedo differences (of $\sim 0.8$ for ice and $>0.2$ for thin ice) between snow-covered ice and open water.
A measure of PC performance or the sensitivity of the system is the number of shots it takes to accumulate a certain number of near-surface or signal photons (an aggregate). Here, we use 100 as the photon count (which can be converted to shots per aggregate, where the aggregate count $=100)$. For MABEL over sea ice, the average varies between $\sim 100$ and 1400 shots per aggregate (see Figs. 3d and 4d), or from 1 photon per shot (near specular surfaces) to 1 photon per 14 shots (low reflectance surfaces). This parameter is fairly stable over snow-covered sea ice ( $\sim 500$ shots per aggregate), but there is considerable variability in thin ice and open water areas (shaded regions in Figs. 3 and 4). Thus, even 
though there are no surface returns from certain shots, the MABEL photon clouds provide a nice depiction of the surface profile of the sea ice surface because of the high pulse repetition rate or small sample spacing $(\sim 4 \mathrm{~cm})$.

\section{c. Elevation distributions}

Here, we examine the elevation distributions of three distinct surface types: lead, lead with specular return, and snow-covered sea ice (Fig. 5). The character of these distributions informs the design of surface elevation retrieval and classification procedures.

In the first example, we show the elevation distribution of a sea ice lead with low signal and background counts (Fig. 5, left panel). The surface and background counts (Fig. 5a), and the associated photon cloud of the $\sim 1.45$-km-wide lead (identified by a horizontal black line in Fig. 5b) are shown within the context of a 13-km MABEL file segment. An exploded view of the photon counts and elevation cloud are shown in Figs. $5 \mathrm{c}$ and 5d, and the elevation distribution of the surface photon cloud can be seen in Fig. 5e. From the 35982 transmitted laser pulses over the $\sim 1.45$-km-wide lead, there were only 1591 surface photons within the $[-2,1] \mathrm{m}$ elevation interval in the neighborhood of the surface. This gives approximately one signal per surface photon in $\sim 22$ shots. From the elevation plot (Fig. 5d, left panel), we see a higher density of the photons below the surface on the right edge of the lead but noticeably higher background counts from the same location. The extended tail in the elevation distribution of the return from the aggregate of 1591 photons (see Fig. 5e, left panel) suggests returns from the subsurface [elevation standard deviation $(\mathrm{SD})=0.40 \mathrm{~m}]$, but it could also be due to surface roughness. As noted below, the returns may also be skewed as a result of the laser pulse shape.

The next example (see Fig. 5, middle panel) shows specular returns from a $\sim 500$-m-wide lead that is flanked by several lower reflectance leads. The signalsurface counts from the specular lead are almost 5 times higher. The background counts from this specular lead, which are comparatively lower than those from adjacent leads, suggest a smooth surface of ice or water. In this case, the signal and solar background counts do not covary because of the specular return from the surface. There are 7470 signal photons in the 12497 shots or $\sim 1$ signal photon in 1.7 shots; this can be contrasted with the 1-in-22 ratio computed for the lead in the previous example. With the larger number of counts from a smooth specular surface over a short distance, the apparent elevation distribution from this photon aggregate (see Fig. 5) provides a measure of the shape of the transmit pulse. The width of the return $(\mathrm{SD}=0.17 \mathrm{~m})$ is narrower than that of the previous example and compares well with the expected pulse width of $2 \mathrm{~ns}(\mathrm{SD}=15 \mathrm{~cm})$ of the laser, which has a sharper leading edge with a trailing edge that decays at a slower rate. In these two examples, we note that this contrast in both elevation and surface/background photon counts (as seen in this and the next example) is extremely useful for the discrimination of ice and open water in freeboard calculations.

The 2-km segment shown in Fig. 5 (right panel) is from a highly reflective snow-covered surface. Both the surface and background counts are consistently higher than those seen in the left and middle panels of Fig. 5. Compared to the photon elevations of the leads in the previous examples, their variability is also higher. In the $1.6-\mathrm{km}$ window, there is $\sim 1$ signal photon in 5 shots, higher than the counts from leads but lower than that from a specular surface. The width of the elevation distribution from the photon aggregate is 0.54 (SD), characteristic of returns from a surface that is rougher than those discussed above. As noted earlier, since the average surface signal per shot from high reflectance surfaces in the current MABEL dataset is less than one, the first photon bias (described in Yang et al. 2011) is zero and does not need to be considered.

\section{Surface elevation retrieval}

In traditional altimetry, the elevation of a surface is estimated from a digitized waveform from the surface scatter. For a PC system, one approach is to create an elevation distribution (or "waveform") from the photon clouds (like those seen in Figs. 5a and 5b) and then use traditional approaches to refine the surface elevation. One advantage of the high PRF and small spot size of PC systems is that there are a number of ways one could select photons to construct these waveforms for surface elevation estimation, which are adapted to the length scale of the surface of interest (e.g., the width of a sea ice lead). In this section, we first describe an approach to retrieve surface elevations in the photon cloud. Second, the key parameters used to control the quality of the retrieval process are discussed. Finally, examples are provided to illustrate the effectiveness of this process with MABEL data.

\section{a. An approach}

We assume that the expected return, $s_{e}(h)$, from a surface with a Gaussian elevation distribution (width $w$ or 2 times the standard deviation) at an elevation offset of $h_{o}$ [i.e., $\left.G\left(h ; h_{o}, w\right)\right]$ can be written as

$$
s_{e}\left(h ; h_{o}, w\right)=s_{t}(h) * G\left(h ; h_{o}, w\right),
$$



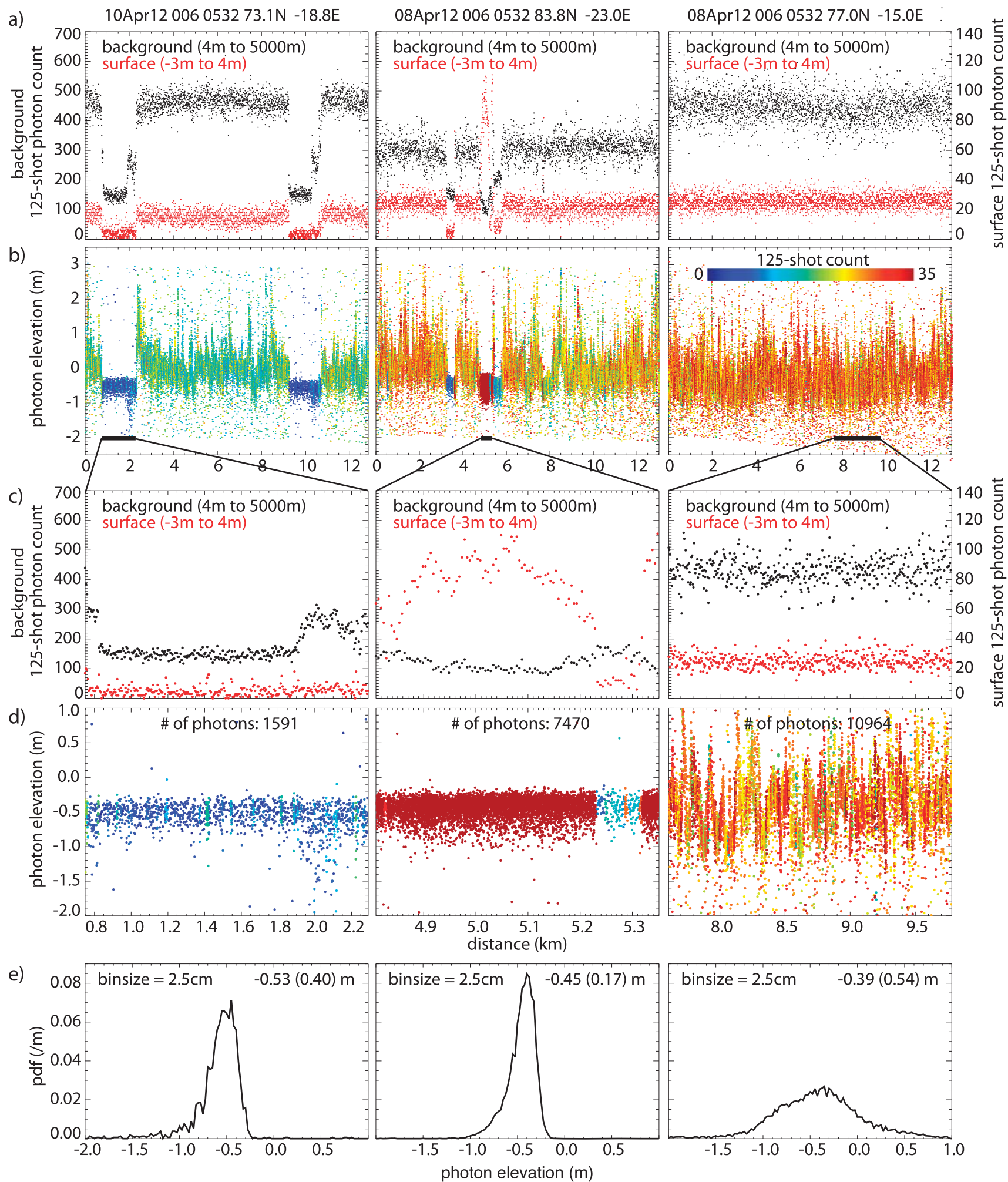

FIG. 5. Elevation distributions of (left) leads, (middle) open water, and (right) snow-covered surfaces (channel 6). (a) Photon counts along a cloud-free MABEL file segment (300 000 shots and $\sim 13 \mathrm{~km}$ long; note the different scales): surface ( -3 to $4 \mathrm{~m})$ and background (4-5000 m) and 125-shot photon counts. (b) Photon elevations; colors represent 125-shot photon counts ( $\sim 5 \mathrm{~m}$ along track). (c) Photon counts in the subsegments indicated by the dark black line in (b). (d) Photon elevations within the subsegments. (e) Elevation distributions within the subsegment (bin size $=2.5 \mathrm{~cm}$ ); quantities in the top-right corners show the distribution means and standard deviations (in parentheses). The plots in the (a),(c) middle panels have the $y$-axis labels of the left and right panels. 
where $s_{t}(h)$ is the impulse response of the system and $*$ is the convolution operator; that is, we assume the expected return to be the convolution of the system impulse response with a Gaussian distribution.

Further, we define the mean squared difference between the normalized received signal (or elevation distribution) $\tilde{S}_{\text {rec }}(h)$ and the normalized expected return, $\tilde{s}_{e}(h)$, to be

$$
\begin{aligned}
e^{2}\left(h_{o}, w\right) & =\frac{1}{N} \sum_{N}\left[\tilde{s}_{\text {rec }}\left(h_{i}\right)-\tilde{s}_{e}\left(h_{i} ; h_{o}, w\right)\right]^{2} \\
\sum_{N} \tilde{s}_{\text {rec }}\left(h_{i}\right) & =1 \\
\sum_{N} \tilde{s}_{e}\left(h_{i} ; h_{o}, w\right) & =1 .
\end{aligned}
$$

The two variables $\tilde{s}_{e}(h)$ and $\tilde{s}_{\text {rec }}(h)$ are normalized because we have no a priori information about the amplitude of the received signals (or the reflectivity of the surface).

Then, we consider the minimum in the error surface, $e^{2}\left(h_{o}, w\right)$, to provide the best estimate of the height offset, $\hat{h}_{o}$, of a surface with a Gaussian distribution of width $\hat{w}$. This can be represented as

$\left(\hat{h}_{o}, \hat{w}\right)=\operatorname{argmin} e^{2}\left(h_{o}, w\right) \quad h_{o} \in\left[h_{1}, h_{2}\right] \quad w \in\left[w_{1}, w_{2}\right] ;$

that is, the location of the given argument for which the function $e^{2}$ attains its minimum value within the intervals defined by $h_{o} \in\left[h_{1}, h_{2}\right]$ and $w \in\left[w_{1}, w_{2}\right]$ is considered the best estimates of $h_{o}$ and $w$.

\section{b. Key parameters in retrieval process}

The key parameters that control behavior of the retrieval process described above are the number of signal photons and bin size used in constructing $\tilde{S}_{\text {rec }}(h)$; the window size or the number of bins [ $N$ in Eq. (3)] of the elevation distribution used in the process; and the size of the search space in width $\left(w \in\left[w_{1}, w_{2}\right]\right)$ and elevation offset $\left(h_{o} \in\left[h_{1}, h_{2}\right]\right)$.

An elevation distribution $\tilde{s}_{\text {rec }}(h)$ can be constructed using photon elevations from a fixed or variable number of shots. Rather than using photons from a fixed number of shots, we find it best to use a fixed number of photons (or aggregates) over a variable number of shots. Over surfaces with lower returns, waveforms contain photons from a larger number of shots, and vice versa. This allows the control of signal strength (or signal-to-noise ratio) of the constructed waveform and thus the consistency in the quality of the surface retrievals. This is well suited for sea ice surfaces with its broad range of expected reflectance. We note, however, that the spatial resolution of the retrievals is proportional to signal strength and varies along track. Below, we show examples of retrieved elevations using different aggregate counts. As for bin size, we use a width of $2.5 \mathrm{~cm}$, which is approximately the elevation resolution of the $\mathrm{PC}$ system.

The window size specifies that a portion of the elevation distribution be used in the surface estimation process. We use the standard deviation $(\sigma)$ of the distribution within -2 to $3 \mathrm{~m}$ of the mean elevation to define the window size. In the results shown here, we truncate the distribution at $2 \sigma$ from the mean; that is, we use a window size of $4 \sigma$. This effectively limits the number of noise photons used in the retrieval process. Potentially, narrowing the width of the window could also be used to reduce the sensitivity of the retrieved elevation to the contributions of subsurface returns present in the tails of the distribution. For the dataset examined, the $[-2,3] \mathrm{m}$ interval works quite well; however, we may have to expand the window to include returns from taller ridges.

To implement our approach, we also require estimates of $s_{t}(h)$, that is, the impulse response of the system. Surface retrievals are sensitive to the shape of $s_{t}(h)$, especially when the surface is relatively flat compared to the pulse width. Asymmetric transmitted pulse shapes or system response, if not accounted for, would introduce biases in the elevation estimates. As measurements of the system impulse response are not available at the time of this writing, we use the elevation distribution of the specular return (Fig. 5, middle panel) in the previous section as a replica of the impulse response pulse. This model of the replica, if not contaminated by subsurface return, suggests asymmetry in the impulse response.

The search space in elevation offset and width seen in Eq. (4) (i.e., $w \in\left[w_{1}, w_{2}\right]$ and $h_{o} \in\left[h_{1}, h_{2}\right]$ ) depends on the quality of the initial estimate of the mean elevation calculated above and the expected distribution of the surface relief. For the results discussed below, the following intervals are used: $h_{o} \in[-0.5,+0.5] \mathrm{m}$, $w \in[0.0,1.5] \mathrm{m}$. The mechanization of the retrieval process is not discussed here, suffice it to say that finding the minimum in $e^{2}\left(h_{o}, w\right)$ can be computationally optimized. Efficiency can be obtained by pregenerating $s_{e}(h)$ over the search space of interest.

\section{c. Retrieval examples}

Here, we discuss the surface retrieval results from a $6.5-\mathrm{km}$ MABEL segment using aggregates of 100 photons to construct the elevation distributions $s_{\text {rec }}$. Figure 6 shows the photon cloud (Fig. 6a), the estimated half-width of the Gaussian $(G)$ (Fig. 6b), and the 


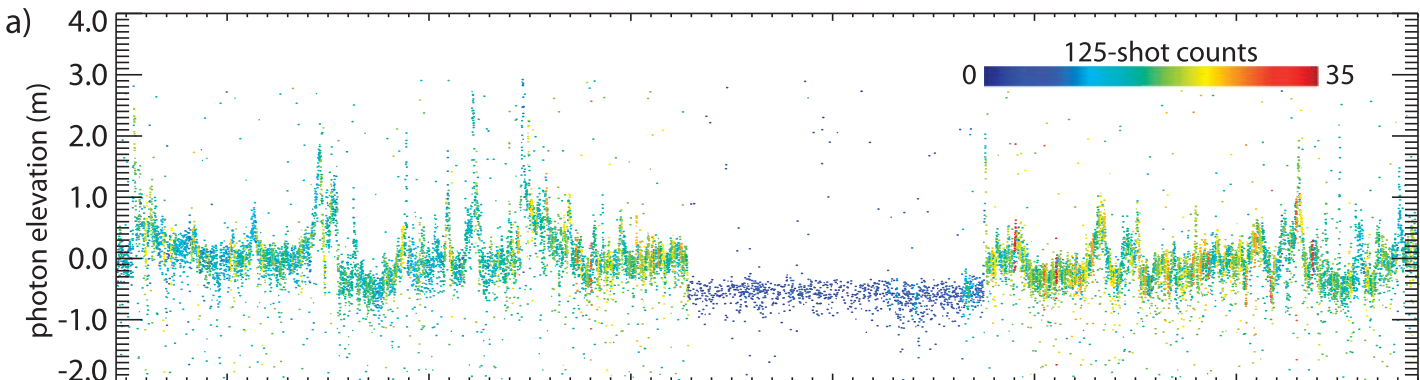

b)
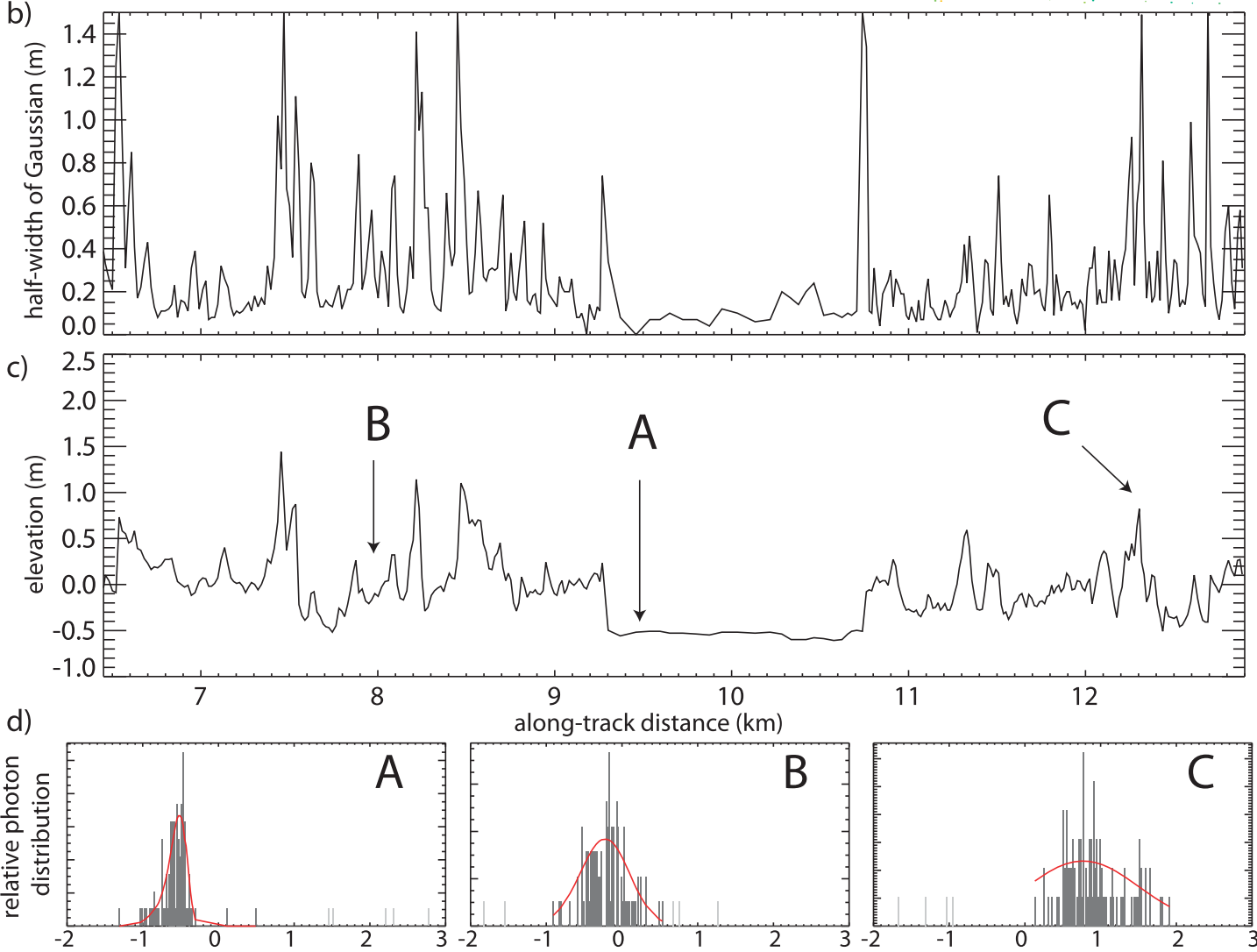

along-track distance $(\mathrm{km})$
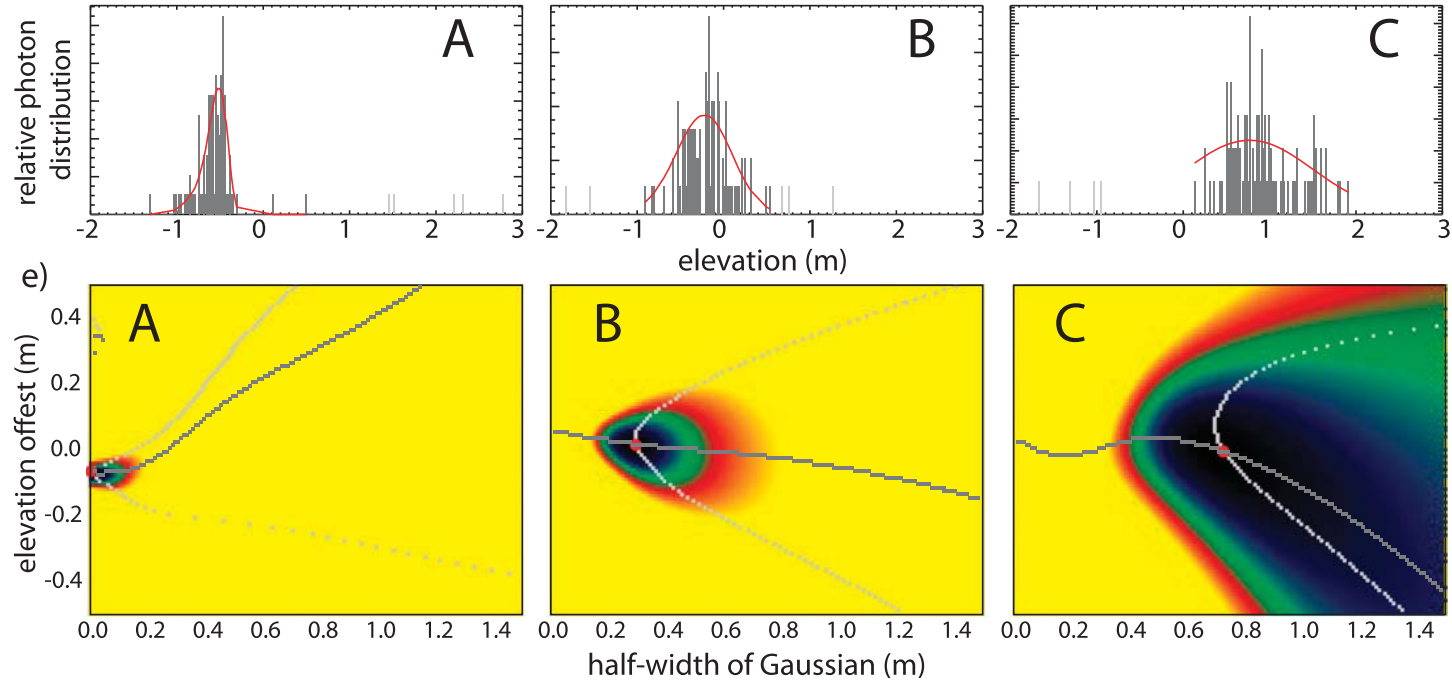

FIG. 6. Surface elevation retrieval using 100-photon aggregates. (a) Photon cloud from a 6.5-km segment of MABEL. (b) Half-width of Gaussian from retrieval process. (c) Retrieved elevation. (d) Elevation distribution and modeled return (red) [at locations A-C in (c)]. (e) Error surface with the light and dark gray lines showing the local minima along the $x$ axis (half-width of Gaussian) and $y$ axis (elevation offset) and with colors showing the relative size of the minima. Panels (a)-(c) have the same $x$ axis as in (c). 
retrieved elevation profile (Fig. 6c). Over a relatively flat portion of the ice cover near the center of the segment (with low photon density-bluish color), the half-width of the Gaussian varies between 0 and $0.3 \mathrm{~m}$, and the elevation varies by $\sim 8 \mathrm{~cm}$. This can be compared to the more deformed (Gaussian half-width ranges up to $1.5 \mathrm{~m}$ ), snow-covered surfaces (of higher photon density) that ranges up to $2 \mathrm{~m}$ in elevation above the lowest point in the profile.

In particular, at three locations (A, B, and C in Fig. 6c) along the MABEL segment, we show the elevation distributions $\left[s_{\mathrm{rec}}(h)\right]$, the modeled return, $\left[s_{e}(h)\right.$ in red], and the error surface $e^{2}\left(h_{o}, w\right)$ used in the estimation of the surface elevations (Figs. 6d and 6e). These locations were selected to illustrate the effectiveness of the retrieval process over a range of roughness scales. The distribution in red can be thought of as the best fit of the modeled returns $\left[s_{e}(h)\right]$ to the $s_{\mathrm{rec}}(h)$.

The $s_{\text {rec }}(h)$ at A (Fig. 6d, left panel) is that of a smooth surface with a relatively narrow distribution that exhibits the asymmetry, with a noticeable tail, that is seen in the specular return in Fig. 5e (left panel), that is, our assumed replica of the system impulse response. The red line in the figure shows the modeled return fitted to the distribution. A very sharp (i.e., the local curvature; Fig. 6e, left panel) and well-defined minimum is evident in $e^{2}\left(h_{o}, w\right)$. The $s_{\text {rec }}(h)$ at B (Fig. $6 \mathrm{~d}$, middle panel) is that of a broader return. In this case, as the surface roughness broadens the return distribution, the expression of the asymmetry in $s_{t}(h)$ is no longer apparent. And, the minimum in $e^{2}\left(h_{o}, w\right)$ (Fig. 6e, middle panel) is not as sharp as that seen in A (Fig. 6e, left panel) but nevertheless clearly identifiable in the search space. The $s_{\text {rec }}(h)$ at C (Fig. 6d, right panel) is that of an even broader return with a bimodal character that suggests photons from a mixture of smooth and deformed ice (perhaps ridges of different heights). Similarly, the expression of the asymmetry in $s_{t}(h)$ is no longer apparent. The minimum in $e^{2}\left(h_{o}, w\right)$ (Fig. 6e, right panel) is broadened further than that seen in A or B. Still, the minimum is evident but the surface is less symmetric about the minimum: the curvature of the surface in the direction that varies the width of the Gaussian is reduced. This is likely due to the mixture of surface roughness in the distribution, as the surface behavior departs from that of the model assumption.

These examples illustrate the importance of the pulse shape in the retrieval of elevation over smooth surfaces. As the elevation distribution deviates from that of a Gaussian when a mixture of ice types are present, the minimum in $e^{2}\left(h_{o}, w\right)$ becomes less pronounced. The curvatures may provide an indicator, in addition to the width of the Gaussian, of the character of the surface.

\section{d. Sensitivity to aggregate count}

As discussed above, the quality and sensitivity of retrieved elevations are sensitive to the size of the photon population (aggregate) used to estimate the mean elevation. Two examples (in Figs. 7 and 8 ) from MABEL illustrate the differences in the retrieval results using aggregates of 50 and 100 photons. Both examples include flat smooth surfaces and rougher snow-covered surfaces. The smoother surfaces are ideal for assessing the relative precision of the instrument and retrieval procedure, and the rough surfaces are useful for understanding the impact of spatial sampling.

The photon clouds in Figs. 7a and 8a show the distribution in elevation as well as the photon densities in elevation (in terms of counts) along the track. There are clear distinctions between lower surface counts from relatively smooth surfaces and higher counts from rougher, snow-covered surfaces. The contrasts between the elevation profiles using 50 and 100 photon aggregates can be seen in Figs. $7 \mathrm{~b}$ and $7 \mathrm{c}$, and $8 \mathrm{~b}$ and $8 \mathrm{c}$. Not unexpectedly, the retrieved elevations using 50-photon aggregates seem more sensitive to surface detail and thus noisier overall. For MABEL, the average spatial resolution of the elevation profile is $\sim 8$ and $\sim 16 \mathrm{~m}$ for the 50- and 100-photon aggregates, respectively; spatial resolution depends on the number of shots needed to accumulate the signal photons used in the aggregate (as indicated in Figs. 7d and 8d).

The second moment (standard deviation) of the surface elevation profiles is useful in assessing the impact of aggregate sizes. In Figs. 7e and 8e, we show the standard deviation of the elevation profile over 100-m intervals. Over relatively flat areas in Fig. 7, we find standard deviations of $\sim(5-6)$ (black) and $2-3 \mathrm{~cm}$ (red) in the 50 and 100-count aggregates, respectively. This suggests that an along-track precision of several centimeters can be obtained with 100-photon aggregates with this particular implementation of the MABEL. Another expected result is that the standard deviation of elevations in the rougher ice is somewhat more muted using the 100-count aggregates because of smoothing of the elevations when larger populations are used. In general, the retrieval process seems well behaved when the aggregate counts are varied.

\section{Comparisons with profiles from the ATM lidar}

On 10 April the MABEL (on ER-2) and ATM lidars (on the P-3 from Operation IceBridge) flew near-coincident ground tracks to obtain surface elevation profiles for assessment of the MABEL surface elevation retrievals. The ATM instrument on the P-3 with an approximate 
a)

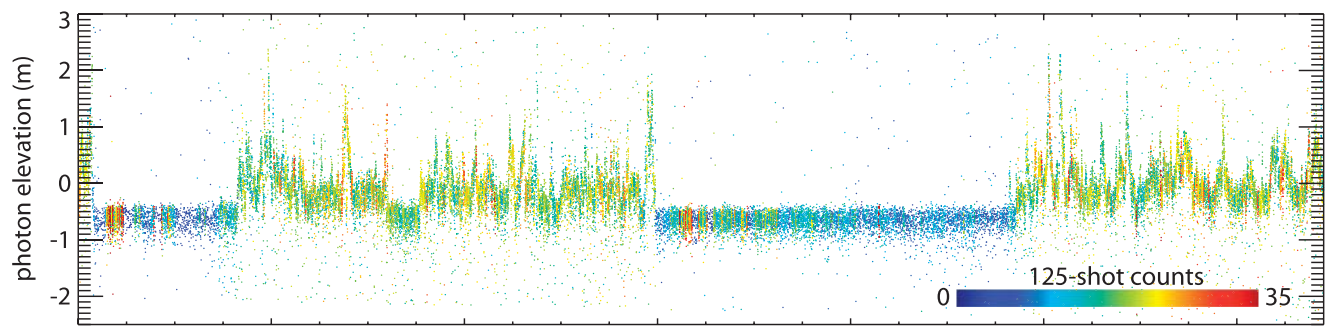

b)

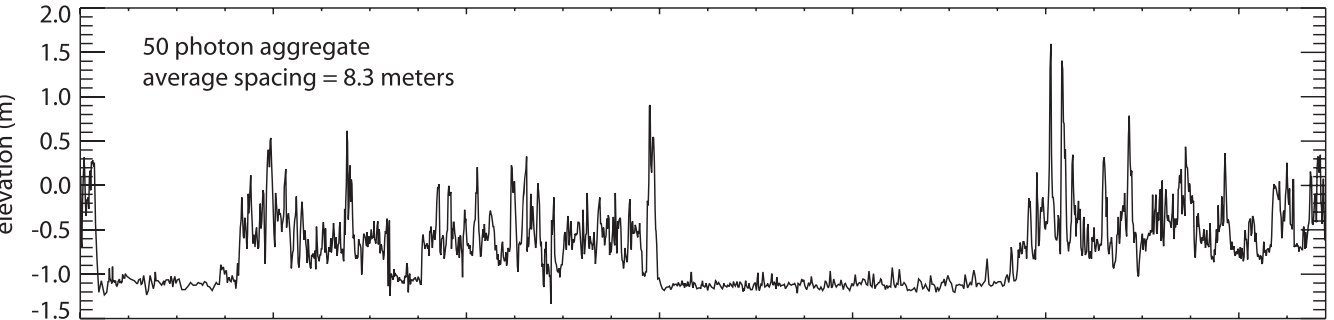

c)
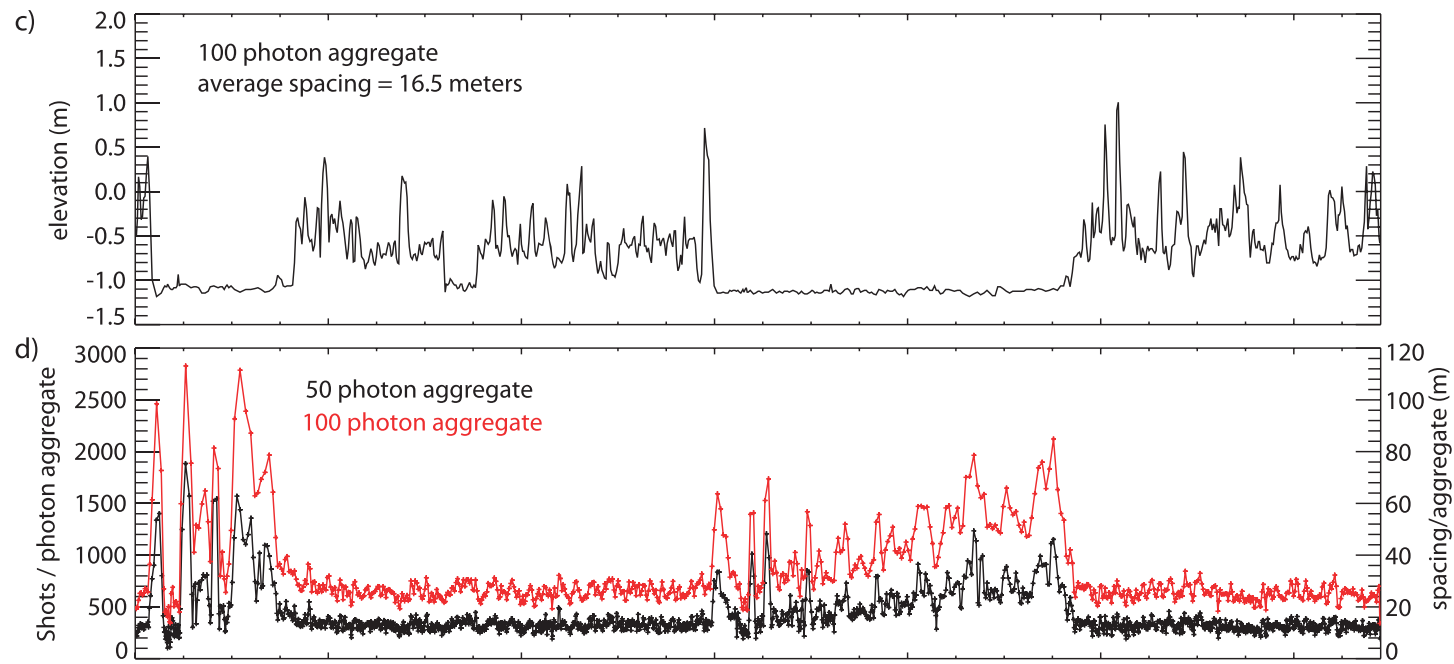

e)

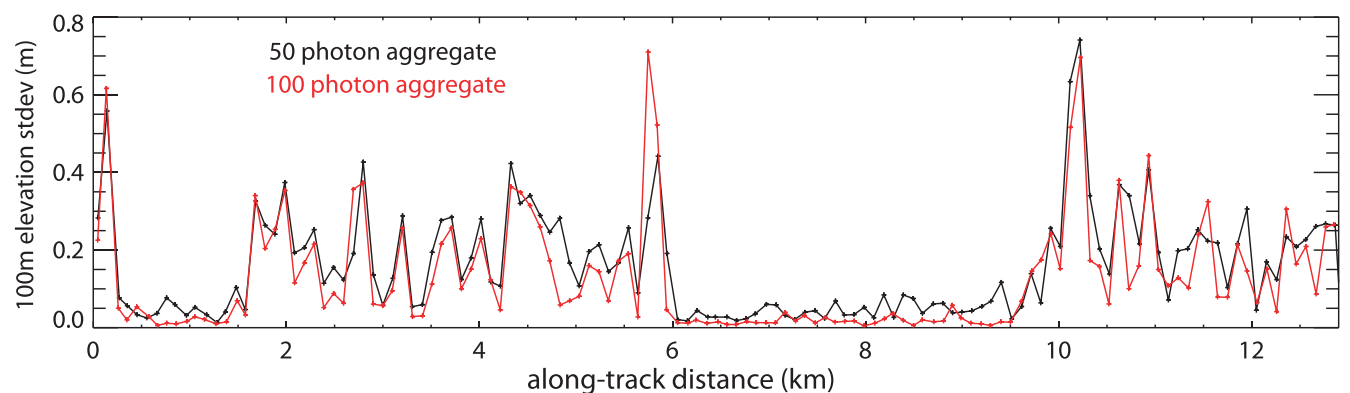

FIG. 7. Example comparison of retrieved surface elevations using 50- and 100-photon aggregates: (a) 125- shot photon counts, (b) 50-photon aggregate elevation, (c) 100-photon aggregate elevation (d) shots and spacing per 50- and 100-photon aggregates, and (e) 100-m elevation standard deviation for 50- and 100-photon aggregates.

scan swath of $\sim 250 \mathrm{~m}$, an analog lidar also at $532 \mathrm{~nm}$, increases the likelihood of coincident coverage (Figs. 9a and $9 b$ ).

The elevation profiles from the two instruments for two different $\sim 4$-km segments are shown in Fig. 9 . These comparisons are produced in two steps: the calculation of average profiles of the two datasets and the location of the MABEL ground track in the ATM swath. Given the approximate resolution of the MABEL surface retrieval $(\sim 15 \mathrm{~m})$, we produce surface elevation estimates from ATM at approximately the same length scale. It should also be noted neither the ATM nor MABEL instruments provide contiguous ground sampling. In the case of ATM, there are gaps associated with the scanning processes and for MABEL there are spots with no surface returns. Elevations for comparison with 
a)

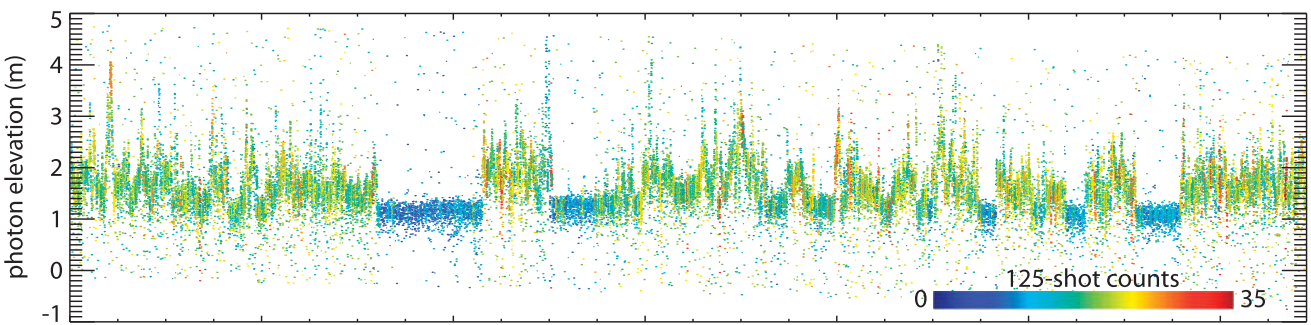

b)

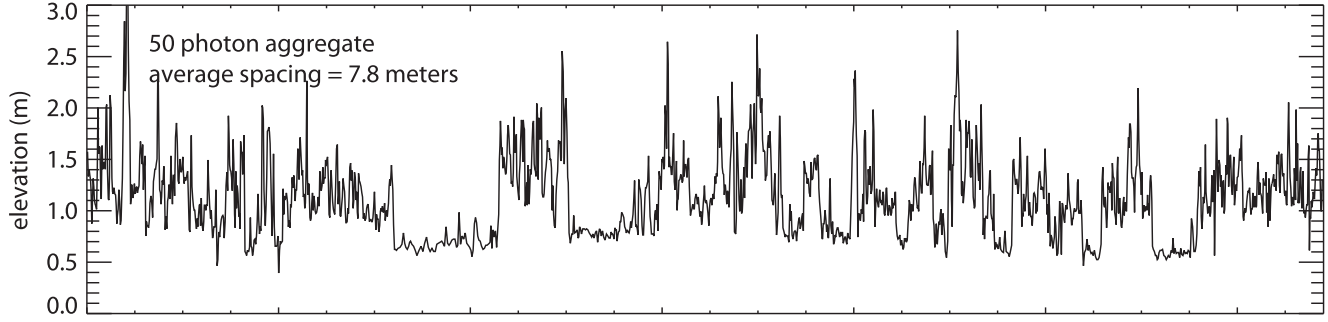

c)

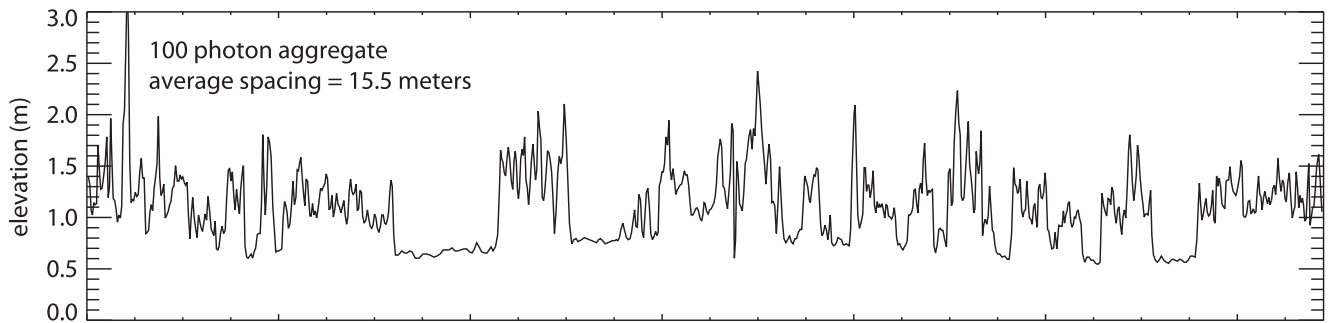

d)

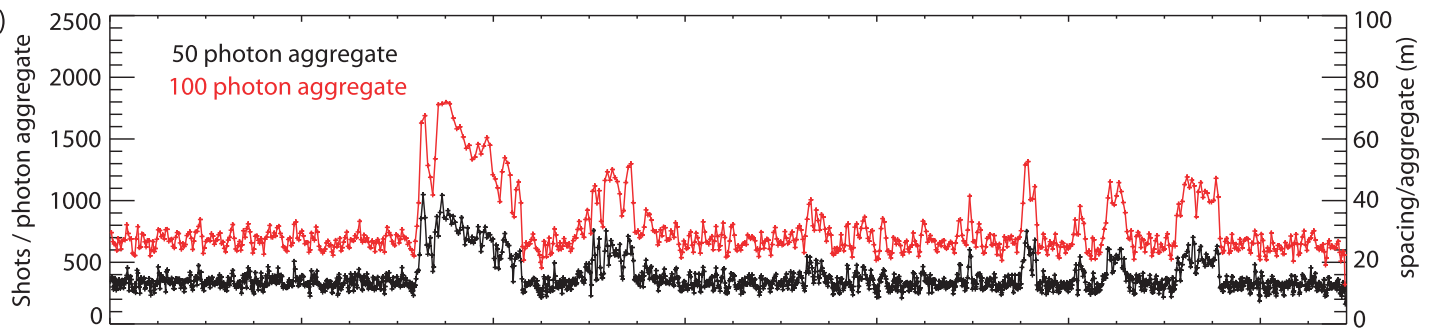

e)

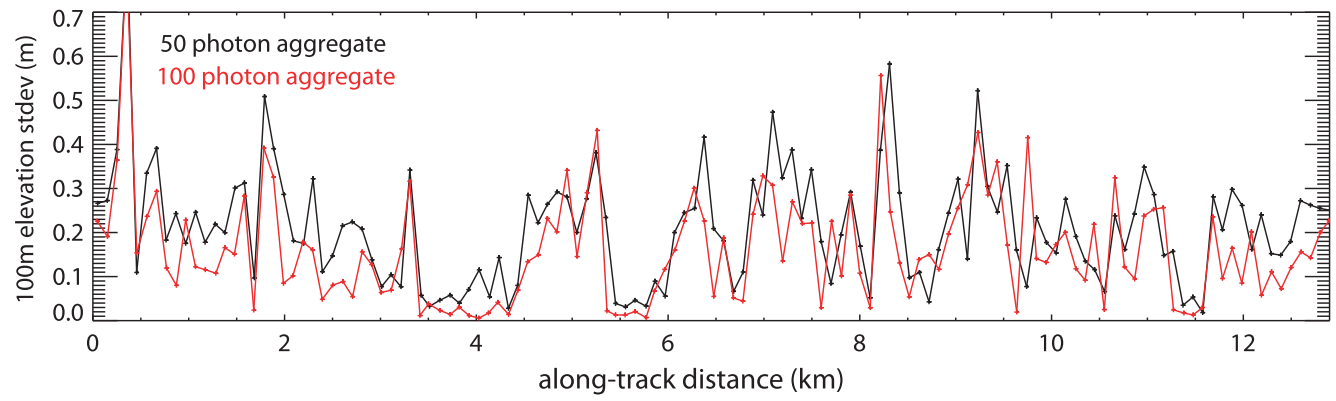

FIG. 8. As in Fig. 7, but for a second example.

MABEL retrievals were calculated by computing $15-\mathrm{m}$ (diameter) Gaussian averages of the ATM elevations centered on each MABEL location.

Accurate collocation of MABEL tracks in the ATM swaths is challenging because of ice drift and the different ground speeds of the instrument platforms (ER-2 and P-3). Ice movements and time delays between acquisitions limit our ability to register finescale surface features along track. As the sea ice moves and deforms, time differences in coverage introduce misregistrations between the surface profiles, especially when movement of discrete ice floes are especially evident at the meterlevel length scales considered here. In the two examples shown in Fig. 9, the delays between the two instruments are $\sim 18 \mathrm{~min}$. The impact of ice motion is appreciable. At a speed of $>10 \mathrm{~cm} \mathrm{~s}^{-1}$ (typical for this area), sea ice drifts $\sim 140 \mathrm{~m}$ in $20 \mathrm{~min}$ but the strain rates (or relative motion between floes) are less predictable. Hence, the expected misregistration between surface features in the presence of surface motion, even with meter-level geolocation accuracy, is not small. The comparisons (in Fig. 9) are produced by maximizing the correlations between the two 



FIG. 9. Comparisons of surface elevation profiles from MABEL with near-coincident ATM profiles. (a) (top) ATM swath with MABEL ground track (black) and (bottom) ATM (red) and MABEL (black) profiles. The time difference shown in the top left corner of the (bottom) panel is between the two airborne platforms. (b) As in (a), but for a different segment along the track. (c),(d) Scatterplot of elevation samples in ATM and MABEL profiles in (a),(b), respectively; numerical values of correlation and differences between the profiles are shown in the top-left corner of each panel; elevations are averages of ATM footprints within $15 \mathrm{~m}$ (diameter) of MABEL locations.

elevation profiles (along and across track) around a best initial location of the MABEL ground track within the ATM swath. As there are residual biases in absolute elevation (relative to the WGS84 reference ellipsoid) in both instruments, the two profiles are tied together (i.e., relative biases removed) at the points indicated by the arrows (in Fig. 9) once the offset with maximum correlation is found.

Even with considerations in spatial coregistration and sampling discussed above, the two comparisons (scatterplots in Fig. 9c) between MABEL and ATM surface profiles nevertheless give correlations of 0.9 and 0.86 with similar standard differences of 0.3 and $0.26 \mathrm{~m}$, respectively. Broadly, the results show that the MABEL and ATM retrievals covary along track and thus are sensitive to similar surface features. The scatter is symmetric around the line with unity slope, which suggests that thickness-dependent biases between the two retrievals are not evident—at least in this dataset. 
However, detailed analyses of the differences may not be not warranted given the contributions of the different error sources discussed above.

\section{Conclusions}

In this paper, surface elevation profiles of the Arctic Ocean sea ice cover acquired by the MABEL instrument are examined. Over the next several years, MABEL will serve as a tool to support the development of algorithms for retrieval of geophysical parameters during the ICESat-2 mission. Since this is the first Arctic deployment of this type of instrument, we provide a broad survey of the phenomenology, one approach to retrieve surface elevation, and an assessment of the retrieval approach. Specifically, this paper addresses the sea ice dataset acquired on 8 and 10 April 2012. Even though damage to the optical fibers during this campaign resulted in degraded performance of the instrument, the acquired dataset provided a valuable first look of the Arctic sea ice cover with a micropulse lidar. In this section, we summarize the results as well as outline future plans on the use of MABEL to provide a broader spectrum of seasonal ice conditions (winter and summer melt) for further evaluation of the profiling capabilities and limitations of the MABEL and ATLAS instruments.

For the ICESat-2 mission, the ATLAS instrument will be tasked to provide basinwide sea ice freeboard estimates for calculation of ice thickness. MABEL will support the demonstration of this requirement to carry out high-precision surface profiling of the sea ice cover with a photon counting (PC) approach. Although there are important distinctions and considerations in dealing with the photon clouds from PC systems, the physical basis of the lidar is not different from that of an analog lidar. We examined the surface signatures of sea ice returns, and the contrasts between the photon distribution and signal strength from different snow-covered sea ice types and various forms of thin ice and open water. Specular returns, from what appears to be thin ice or open water, are particularly distinctive in the MABEL returns. Subsurface or rough surface returns seem to be present in the photon clouds in some of the MABEL file segments over sea ice leads. But, the unambiguous identification and likelihood of such occurrences are more difficult to quantify. This remains a topic of investigation with upcoming MABEL data acquisitions.

An approach to retrieve surface elevations in the observed photon cloud was introduced. Elevation distributions of the surface are constructed using a fixed number of photons (or aggregates) over a variable number of shots and then compared with modeled returns. Fixed-sized aggregates allow the control of signal strength and the consistency in the quality of the surface retrievals. Returns are modeled as the convolution of the system impulse response with that of a Gaussian distributed surface elevation distribution. This is well suited for sea ice surfaces with its broad range of expected reflectance. Restricting the width of the window used in the surface analysis can mitigate potential biases in the elevation estimates due to subsurface returns at $532 \mathrm{~nm}$. At this writing, the impact of subsurface return on elevation retrievals awaits the analysis of larger volumes of MABEL data from future acquisitions. Retrieved surface elevations over relative flat sea ice leads suggest that precisions of several centimeters seem attainable.

On 10 April, the MABEL (on ER-2) and ATM lidars [on the P-3 from Operation IceBridge (Koenig et al. 2010)] flew near-coincident ground tracks to obtain surface elevation profiles for assessment of surface elevation retrievals from MABEL. The two comparisons between MABEL and ATM surface profiles gave correlations of 0.9 and 0.86 with similar standard differences of 0.3 and $0.26 \mathrm{~m}$, respectively. The results show that the MABEL and ATM retrievals covary alongtrack and thus are sensitive to similar surface features. However, detailed assessment of the differences in this particular dataset may not be warranted given the contributions of the different error sources: resolution, sampling, registration, ice motion, and the variability of surface relief at the 10-15-m length scale.

Over the next several years, MABEL will be tasked to provide data to support development of operational algorithms for retrieval of geophysical parameters. During the ICESat-2 mission, the ATLAS instrument will acquire year-round data over the ice-covered ocean of the Arctic and Antarctic, which includes all types of surface and atmospheric conditions during different seasons and through seasonal transitions. The sensitivity of surface retrievals to solar background noise and atmospheric effects such as signal reduction through absorption and scattering as well as multiple-scattering-induced range delay must be characterized. Understanding these geophysical and system characteristics is crucial for the design of robust retrieval algorithms. The goal, for MABEL, is to provide a broad spectrum of seasonal ice conditions (melt and summer) for further evaluation of the profiling capabilities and limitations of the MABEL and ATLAS instruments. Additionally, the issue of subsurface returns at $532 \mathrm{~nm}$ on surface elevation retrieval remains. At this writing, the next deployment of MABEL will be to acquire data over the summer ice cover of the Arctic Ocean. 
Acknowledgments. The digital camera system (DCS) on the ER-2 is operated and maintained by the Airborne Sensor Facility under the auspices of NASA's Airborne Science Program. RK and GFC carried out this work at the Jet Propulsion Laboratory, California Institute of Technology, under contract with the National Aeronautics and Space Administration.

\section{REFERENCES}

Abdalati, W., and Coauthors, 2010: The ICESat-2 laser altimetry mission. Proc. IEEE, 98, 735-751, doi:10.1109/ JPROC.2009.2034765.

Abshire, J. B., X. Sun, H. Riris, J. M. Sirota, J. F. McGarry, S. Palm, D. Yi, and P. Liiva, 2005: Geoscience Laser Altimeter System (GLAS) on the ICESat mission: On-orbit measurement performance. Geophys. Res. Lett., 32, L21S02, doi:10.1029/ 2005GL024028.

Grenfell, T. C., and D. K. Perovich, 1984: Spectral albedos of sea ice and incident solar irradiance in the southern Beaufort Sea. J. Geophys. Res., 89, 3573-3580, doi:10.1029/JC089iC03p03573.

Harding, D., P. Dabney, S. Valett, A. Yu, A. Vasilyev, and A. Kelly, 2011: Airborne polarimetric, two-color laser altimeter measurements of lake ice cover: A pathfinder for NASA's ICESat-2 spaceflight mission. 2011 IEEE Int. Geoscience Remote Sensing Symp. (IGARSS), Vancouver, BC, Canada, IEEE, 3598-3601, doi:10.1109/IGARSS.2011.6050002.

Koenig, L., S. Martin, M. Studinger, and J. Sonntag, 2010: Polar airborne observations fill gap in satellite data. Eos, Trans. Amer. Geophys. Union, 91, 333-334, doi:10.1029/ 2010EO380002.
Krabill, W. B., and Coauthors, 2002: Aircraft laser altimetry measurement of elevation changes of the Greenland ice sheet: Technique and accuracy assessment. J. Geodyn., 34, 357-376, doi:10.1016/S0264-3707(02)00040-6.

Kwok, R., G. F. Cunningham, M. Wensnahan, I. Rigor, H. J. Zwally, and D. Yi, 2009: Thinning and volume loss of the Arctic Ocean sea ice cover: 2003-2008. J. Geophys. Res., 114, C07005, doi:10.1029/2009JC005312.

McGill, M. J., D. L. Hlavka, W. D. Hart, V. S. Scott, J. D. Spinhirne, and B. Schmid, 2002: Cloud physics lidar: Instrument description and initial measurement results. Appl. Opt., 41, 3725-3734, doi:10.1364/AO.41.003725.

—, T. Markus, V. S. Scott, and T. Neumann, 2013: The Multiple Altimeter Beam Experimental Lidar (MABEL): An airborne simulator for the ICESat-2 mission. J. Atmos. Oceanic Technol., 30, 345-352, doi:10.1175/JTECH-D-12-00076.1.

Perovich, D. K., T. C. Grenfell, B. Light, and P. V. Hobbs, 2002: Seasonal evolution of the albedo of multiyear Arctic sea ice. J. Geophys. Res., 107, 8044, doi:10.1029/2000JC000438.

Schutz, B. E., H. J. Zwally, C. A. Shuman, D. Hancock, and J. P. DiMarzio, 2005: Overview of the ICESat mission. Geophys. Res. Lett., 32, L21S01, doi:10.1029/2005GL024009.

Spinhirne, J. D., 1993: Micro pulse lidar. IEEE Trans. Geosci. Remote Sens., 31, 48-55, doi:10.1109/36.210443.

Yang, Y., A. Marshak, S. P. Palm, T. Varanai, and W. J. Wiscombe, 2011: Cloud impact on surface altimetry from a spaceborne 532-nm micropulse photon-counting lidar: System modeling for cloudy and clear atmospheres. IEEE Trans. Geosci. Remote Sens., 49, 4910-4919, doi:10.1109/TGRS.2011.2153860.

Zwally, H. J., and Coauthors, 2011: Greenland ice sheet mass balance: Distribution of increased mass loss with climate warming; 2003-07 versus 1992-02. J. Glaciol., 57, 88-102, doi:10.3189/002214311795306682. 